\title{
A Retrospective Study on Role of DTI in Brain Tumours
}

\author{
G. Karthika ${ }^{1}$, Anand Rajamani ${ }^{2}$, Vayshak K.V. ${ }^{3}$, S. Sowmiya ${ }^{4}$ \\ ${ }^{1}$ Post Graduate, Department of Radiology, Chettinad Hospital and Research Institute, ${ }^{2}$ Assistant Professor, Department of \\ Radiology, Chettinad Hospital and Research Institute, ${ }^{3}$ M.sc Radiology, Department of Radiology, Chettinad Hospital and \\ Research Institute, ${ }^{4}$ Post Graduate, Department of Radiology, Chettinad Hospital and Research Institute, India
}

Corresponding author: Dr. Anand Rajamani, Flat No 1 Phase 1, No 76 BBC City Park, Anna Salai, Chinna Porur, Chennai 600116, India

DOI: http://dx.doi.org/10.21276/ijcmsr.2019.4.4.4

How to cite this article: G. Karthika, Anand Rajamani, Vayshak K.V., S. Sowmiya. A retrospective study on role of DTI in brain tumours. International Journal of Contemporary Medicine Surgery and Radiology. 2019;4(4):D14-D18.

\section{A B S T R A C T}

Introduction: Brain tumors are a heterogeneous group of benign and malignant tumors. Preoperative radiological assessment is vital in understanding the extent of disease invasion and planning surgical management. This study is to analyze the role of DTI in assessing the different patterns of white matter involvement in patients with brain tumors.

Material and methods: A retrospective study was conducted in thirty patients with brain tumours who had undergone both conventional and diffusion tensor imaging. Based on the imaging, the white matter tracts surrounding the tumours were characterized as either displaced or infiltrated. All the medical records of the people diagnosed with brain tumors between February 2018 to February 2019 were reviewed.

Results: A total of 30 subjects were included. The proportion of subjects with low grade and high-grade gliomas was $53.3 \%$ and $46.7 \%$ respectively. Diffusion tensor imaging had shown invasion in 17 (56.7\%) of the subjects. Total resection was done in $17(56.7 \%)$ of the subjects and the remaining 13 (43.3\%) underwent subtotal resection. The incidence of all the postoperative complications was higher among subjects with invasion shown in DTI, but none of the differences have shown statistically significant differences.

Conclusion: Diffusion tensor imaging is a very useful tool in the pre-operative assessment of white matter involvement in cerebral tumors. The people with invasion shown in pre-operative assessment are more likely to have post-operative neurological deficit.

Keywords: DTI, Brain Tumours

\section{INTRODUCTION}

Brain tumors are a heterogeneous group of benign and malignant tumors either primarily originated from brain parenchyma and its surrounding structures or secondary to primary tumors in other parts of the body. A recent metaanalysis has estimated incidence rate of all brain tumors to be 10.82 (95\% CI: 8.63-13.56) per 100000 person-years. ${ }^{1}$ As per recent statistics from Global burden of disease study, in 2016, there were 330000 incident cases of CNS cancer and 227000 deaths globally, with 17.3\% increase in agestandardised incidence rate globally. ${ }^{2}$ Brian tumors pose a significant diagnostic and therapeutic challenge to the treating clinician, often leading to delayed diagnosis and related adverse consequences. ${ }^{3}$

Pre-operative neuro imaging plays a vital role in not only identification, but also in clear anatomical demarcation, in reasonably estimating the possible pathological type of tumor and planning for surgery. ${ }^{4}$ The most important aspect of this preoperative assessment is clearly demarcating the involvement of surrounding white matter tracts, to allow for complete tumor resection, with minimal damage to surrounding healthy tissue. ${ }^{5}$ Traditional neuroimaging methods, Conventional MRI methods are not very efficient in this aspect. Diffusion-tensor imaging with tractography (DTI), which has been reported to be highly superior than conventional MRI in diagnosis of brain tumors. ${ }^{6-8}$

Diffusion-tensor imaging with tractography (DTI) imaging provides a presurgical and intra operative tool, that in conjunction with other modalities, enables a safer, more patient-specific procedure. ${ }^{9,10}$ DTI-merged functional neuronavigation could be a useful tool in brain tumor surgery for maximal safe resection. ${ }^{11,12}$ Apart from the advantages associated with DTI, there are limitations concerned with DTI, such as lack of gold standard technique available to validate tractography and its reliability. The resolution of DTI may be insufficient which may lead to substantial partial volume effect. Greater sensitivity for movements, Susceptibility to produce artefacts as a result of echo planar imaging method, etc are the other limitations. Many of the limitations can be overcome via advanced magnetic field, powerful and quicker gradients, non-echo planar imaging sequences.

Documenting the role of DTI and its limitations in a particular setting may provide vital inputs to the clinicians in optimal utilization of the procedure. But there is relative scarcity of studies in Indian population documenting the role of DTI in diagnosis of brain tumors. The current study 
was aimed at fulfilling this knowledge gap and add to the available evidence on role of DTI in brain tumors.

\section{MATERIAL AND METHODS}

A retrospective cohort study was conducted in the Department of Radiology, Chettinad hospital and Research Institute, Chennai. All the medical records of the people diagnosed with brain tumors between February 2018 to February 2019 were reviewed. 30 patients (24 male and 6 female) with intracranial neoplasms who underwent MRI for presurgical evaluation, were included in the study. The study included both low grade and high grade gliomas. All patients signed a term of free and informed consent. The study was approved by the Ethics Committee(Research) of the institute.

\section{MRI acquisition}

A baseline MRI brain was obtained from all the patients in 1.5 Tesla SIGNA GE HDxt MRI scanner using a 8 channel NV(NAVIGATOR) radiofrequency coil. The bore size of machine is $60 \mathrm{~cm}$.

T1 and T2 weighted images were taken. Additional sequences such as fluid attenuation inversion recovery, diffusion weighted image and apparent diffusion coefficient sequences were obtained. Post contrast T1 $\mathrm{W}$ fat saturated sequences were also obtained. DTI was done subsequently.

\section{DTI acquisition}

DTI was performed using a single-shot spin-echo echoplanar-imaging pulse sequence with a diffusion sensitization $\left(b=1000\right.$ seconds $\left./ \mathrm{mm}^{2}\right)$, TR $3000 \mathrm{~ms}$, TE $94 \mathrm{~ms}$, slice thickness between $2.5 \mathrm{~mm}$, no gap between slices, matrix $\times$ $92 \times 88$, flip angle $90^{\circ}$.

\section{Diffusion tensor imaging tractography}

Images were then analyzed using DTI Studio, obtaining main eigenvector, and fractional anisotropy (FA) maps. From their combination, color maps were generated with conventional color-coding. Deterministic tractography was performed in all patients to reconstruct subcortical connections using the fiber assignment by the continuous tracking method. An FA threshold of 0.1 and a turning angle 55.8 were used as criteria to start and stop tracking. The CST and some of the major subcortical tracts were reconstructed, defining for each tract separately regions of interest (ROIs) around areas of WM that all the fibers of each tract must pass through to reach their cortical or subcortical end stations. DTI tractography images were systematically reviewed. Tracts were then classified as Displaced, Infiltrated, and Disrupted.

Displaced: If the tract showed normal or only slightly decreased FA, with abnormal location and/or direction, resulting from bulk mass displacement. Infiltrated: if the tract showed reduced anisotropy but remained identifiable on color maps. Disrupted: if the tract showed isotropic (or near-isotropic) diffusion, such that it could not be identified on directional color maps.

\section{RESULTS}

The male to female ratio in the study population was $4: 1$. The number of subjects with the age $\leq 45$ years was 14 (46.7\%), the remaining $16(53.3 \%)$ of the subjects were above 45 years. Pre-operative neurological assessment showed motor weakness in 16 (53.3\%) of the subjects. Seizures were found in 14 (46.7\%) of the subjects. The other common neurological defects were memory impairment and urinary incontinence in 5(16.7\%) and 4(13.3\%) subjects each. (Table 1)

The proportion of subjects with low grade and high-grade gliomas was $53.3 \%$ and $46.7 \%$ respectively. DTI had shown invasion in 17 (56.7\%) of the subjects. Total resection was

\begin{tabular}{|l|l|c|}
\hline Characteristics & Total no of patients(n=30) & Number of cases \\
\hline Sex & Male & $24(80 \%)$ \\
& Female & $6(20 \%)$ \\
\hline Age & $\leq 45$ years & $14(46.7 \%)$ \\
& $>45$ years & $16(53.3 \%)$ \\
\hline Pre-operative & Motor weakness & $16(53.3 \%)$ \\
neurological assessment & Memory impairment & $5(16.7 \%)$ \\
& Seizure & $14(46.7 \%)$ \\
\hline \multicolumn{2}{|c|}{ Table-1: Demographic characteristics and neurological status among the study population } \\
\hline
\end{tabular}

\begin{tabular}{|l|l|c|}
\hline Characteristics & Total no of patients(n=30) & Number of cases \\
\hline Grade of tumour & Low grade glioma & $16(53.3 \%)$ \\
& High grade glioma & $14(46.7 \%)$ \\
\hline DTI & Displaced only & $18(60 \%)$ \\
& Invaded & $12(40 \%)$ \\
\hline Surgery & Subtotal resection & $13(43.3 \%)$ \\
& Total resection & $17(56.7 \%)$ \\
\hline Post-operative & Power deterioration & $5(16 \%)$ \\
neurological assessment & Power improvement & $6(20 \%)$ \\
& Seizure & $3(10 \%)$ \\
& Memory impairment & $6(20 \%)$ \\
\hline \multicolumn{2}{|c|}{ Table-2: Tumor characteristics, type of surgery and post-operative functional status } \\
\hline
\end{tabular}




\begin{tabular}{|c|c|c|c|c|}
\hline \multirow[t]{2}{*}{ Post-operative outcomes } & \multicolumn{2}{|c|}{ DTI } & \multirow[t]{2}{*}{ Chi square } & \multirow[t]{2}{*}{ Fisher exact $P$ value } \\
\hline & Invaded ( $\mathrm{N}=12$ ) & Displaced ( $N=18)$ & & \\
\hline \multicolumn{5}{|l|}{ Power Detoriation } \\
\hline Present & $4(33.33 \%)$ & $2(11.11 \%)$ & \multirow[t]{2}{*}{2.222} & \multirow[t]{2}{*}{0.184} \\
\hline Absent & $8(66.67 \%)$ & $16(88.89 \%)$ & & \\
\hline \multicolumn{5}{|l|}{ Power Improvement } \\
\hline Present & $1(8.33 \%)$ & $5(27.78 \%)$ & \multirow[t]{2}{*}{1.701} & \multirow[t]{2}{*}{0.358} \\
\hline Absent & $11(91.67 \%)$ & $13(72.22 \%)$ & & \\
\hline \multicolumn{5}{|l|}{ Memory Impairment } \\
\hline Present & $4(33.33 \%)$ & $2(11.11 \%)$ & \multirow[t]{2}{*}{2.222} & \multirow[t]{2}{*}{0.184} \\
\hline Absent & $8(66.67 \%)$ & $16(88.89 \%)$ & & \\
\hline \multicolumn{5}{|l|}{ seizure } \\
\hline Present & $2(16.67 \%)$ & $1(5.56 \%)$ & \multirow[t]{2}{*}{0.988} & \multirow[t]{2}{*}{0.548} \\
\hline Absent & $10(83.33 \%)$ & $17(94.44 \%)$ & & \\
\hline \multicolumn{5}{|l|}{ Urinary Incontinence } \\
\hline Present & $1(8.33 \%)$ & $0(0 \%)$ & \multirow{2}{*}{\multicolumn{2}{|c|}{ *No statistical test was applied- due to 0 subjects in the cells }} \\
\hline Absent & $11(91.67 \%)$ & $18(100 \%)$ & & \\
\hline
\end{tabular}

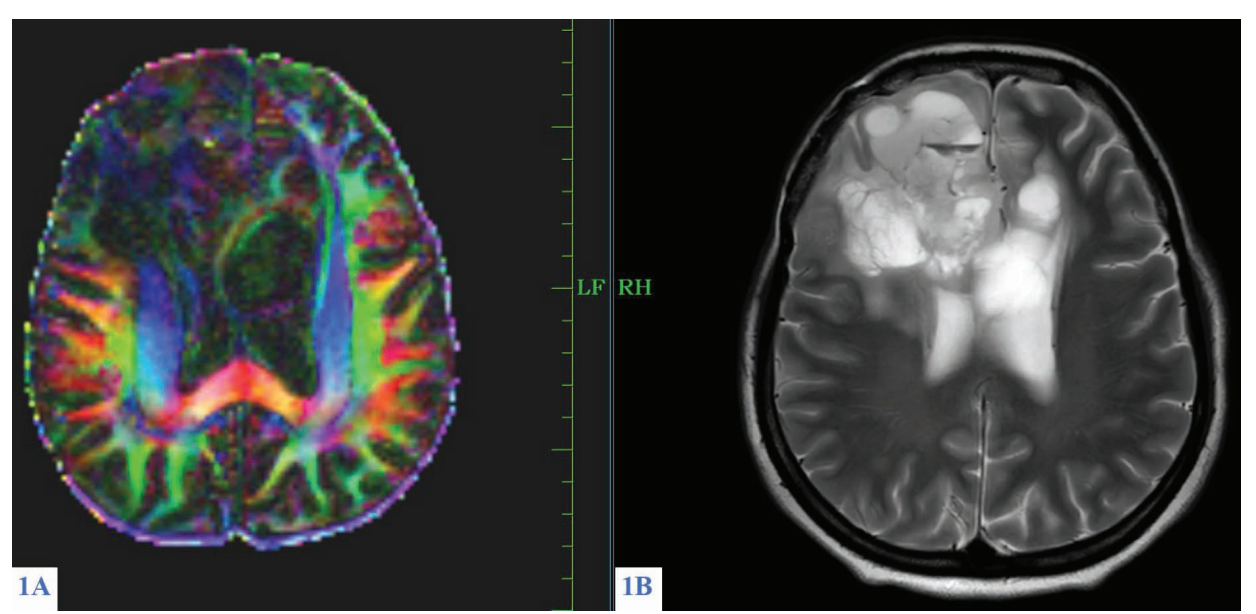

Figure-1: A 36 year old female with glioblastoma multiforme involving the bilateral frontal lobes and corpus callosum (butterfly pattern).

1A. Axial directional color map showing complete destruction of right superior longitutional fibers and the genu of corpus callosum.

1B. Axial T2 weighted image shows heterogenous mass involving bilateral frontal lobes and genu of corpus callosum with surrounding perilesional edema.

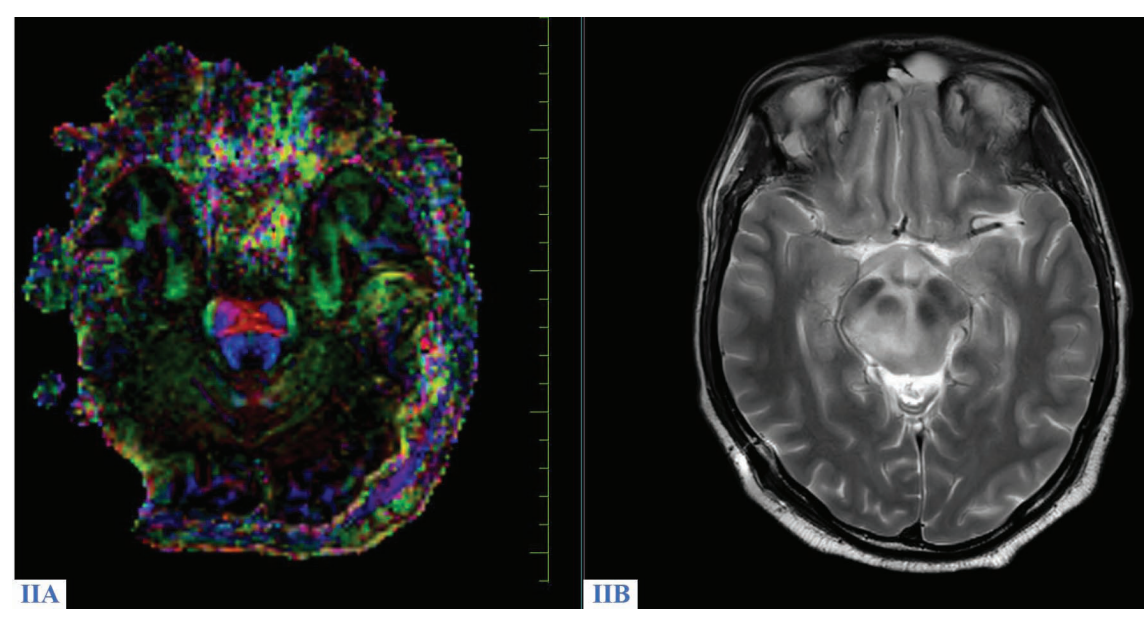

Figure-2: An 18 yr old male with diffuse midline (brainstem) glioma.

IIA. Axial directional color map showing loss of fibers involving the right cortico-spinal tract at midbrain level.

IIB. Axial T2 weighted image hyperintense mass lesion involving the midbrain predominantely on the right side. 


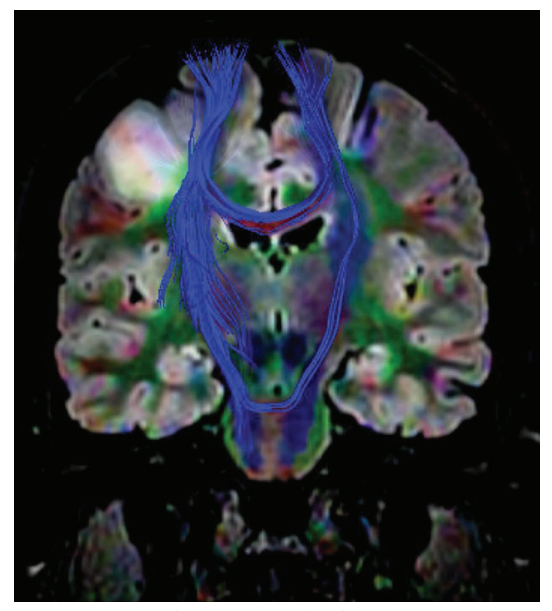

Figure-3: Coronal diffusion tensor imaging of a 22 year old male with right frontal ganglioglioma showing mild displacement of the right corticospinal tract.

done in $17(56.7 \%)$ of the subjects and the remaining 13 (43.3\%) underwent subtotal resection. Post-operative power deterioration and improvement were seen in 5(16\%) and 6(20\%) subjects each. Memory impairment was seen in 20\% of the subjects. Seizure and urinary incontinence were seen in 3(10\%) and 1 subject. (Table 2)

The proportion of people with power deterioration in the postoperative period was higher among the subjects among the invaded group, as compared to non-invaded group (33.33\% vs $11.11 \%, P$ value 0.184 ), but the difference was statistically not significant. The proportion of people with memory Impairment in the post-operative period was higher among the subjects among the invaded group, as compared to noninvaded group (33.33\% vs $11.11 \%$, P value 0.184 ), but the difference was statistically not significant. The proportion of people with seizures in the post-operative period was higher among the subjects among the invaded group, as compared to non-invaded group $(16.67 \% \%$ vs $5.56 \%$, $\mathrm{P}$ value 0.548$)$, but the difference was statistically not significant. Only 1 subject in invaded group had urinary incontinence in the post-operative period. Lower proportion of subjects in Non invaded group had shown power improvement in the postoperative period ( $8.33 \% \mathrm{Vs} 27.78 \%$, P value 0.358). (Table 3)

\section{DISCUSSION}

In this retrospective study, 30 patients with brain tumours have been included and quantitative assessment of DTI metrics in white matter tract was performed. The qualitative assessment of fibre tracts was performed in brain tumour cases.

Fibre tractography is a novel MR technique, with this it is made possible to relatively predict the specific tract involved in brain tumours. ${ }^{13}$ The white matter tracking is based on the direction of fibres and the anatomical location. Colour coded maps are generated from the tensor asset images and each white matter fibre tract is assigned a specific colour based on the direction of its fibres. We observed that in our study the corticospinal tract was the most common tract to be affected. Surgery plays a pivotal role in the treatment of intracranial tumours. The extent of resection is a major factor that affects long-term survival in brain tumour patients and this can largely be determined on the basis of the proximity of the lesion to eloquent brain regions. DTI is a technique which is increasingly used now to outline eloquent brain regions prior to surgery.

The vital role DTI can play in intracranial tumours is preoperative confirmation of integrity and location of displaced WM tracts. WM tracts may be pathologically altered by the tumour in several ways. They may be displaced, infiltrated by tumour and/or oedema, or destroyed. ${ }^{14}$ When we compare it with conventional MRI which provides anatomical information, DTI provides crucial information on central nervous system connectivity by enabling precise imaging of the white matter tracts and thus provide guidance during resection of the tumour. ${ }^{5}$

Many previous studies it was concluded that high grade gliomas were significantly associated with infiltration of the white matter tracts and a similar pattern was observed in our study also. ${ }^{13,15}$ Also the extent of resection was related to white matter tract involvement and that complete resection was achievable only when white matter tracts were displaced. In patients, where the white matter tracts were infiltrated sub-total resection only was possible. ${ }^{16}$

This study has shown that DTI can be a very important tool for the preoperative planning of surgery. The involvement of white matter tract is associated with surgical outcome. People with involvement of white matter tract in post-operative DTI had higher probability of post-operative complications. This can be attributed to probability of total resection when white matter tracts are intact and conversely the expected probability of total resection is substantially lower in the cases in which tractography showed infiltrated or displaced WM tracts. Thus, DTI could be postulated as a useful tool to aid in surgical decision making.

\section{Limitations of DTI}

Apart from the advantages associates with DTI, there are limitations concerned with DTI, such as

There is no gold standard technique available to validate tractography and its reliability.

The resolution of DTI may be insufficient which may lead to substantial partial volume effect.

Greater sensitivity for movements

Susceptibility artefacts may be produced as a result of echo planar imaging method.

Many of the limitations can be overcome via advanced magnetic field, powerful and quicker gradients, non-echo planar imaging sequences.

\section{CONCLUSION}

DTI is a very useful tool in pre-operative assessment of white matter tracts involvement in cerebral tumors. Even though DTI has a lot of limitations, this technique has become an important tool in the preoperative assessment of brain tumours close to eloquent areas, there by optimizing surgical strategies. The people with invasion shown in preoperative assessment are more likely to have post-operative neurological deficit.

\section{REFERENCES}

1. de Robles P, Fiest KM, Frolkis AD, Pringsheim T, 
Atta C, St Germaine-Smith C, et al. The worldwide incidence and prevalence of primary brain tumors: a systematic review and meta-analysis. Neuro Oncol. 2015;17(6):776-83.

2. Global, regional, and national burden of brain and other CNS cancer, 1990-2016: a systematic analysis for the Global Burden of Disease Study 2016. Lancet Neurol. 2019;18(4):376-93.

3. Walter FM, Penfold C, Joannides A, Saji S, Johnson M, Watts $\mathrm{C}$, et al.Missed opportunities for diagnosing brain tumours in primary care: a qualitative study of patient experiences. Br J Gen Pract. 2019;69(681):e224-e35.

4. Arbizu J, Dominguez PD, Diez-Valle R, Vigil C, Garcia-Eulate R, Zubieta JL, et al. [Neuroimaging in brain tumors]. Rev Esp Med Nucl. 2011;30(1):47-65.

5. Alexopoulos G, Cikla U, E1 Tecle N, Kulkarni N, Pierson M, Mercier P, et al. The value of white matter tractography by diffusion tensor imaging in altering a neurosurgeon's operative plan. World Neurosurg. 2019.

6. Shaikh S, Kumar A, Bansal A. Diffusion tensor imaging: An overview. Neurol India. 2018;66(6):1603-11.

7. Pujol S, Wells W, Pierpaoli C, Brun C, Gee J, Cheng $\mathrm{G}$, et al. The DTI Challenge: Toward Standardized Evaluation of Diffusion Tensor Imaging Tractography for Neurosurgery. J Neuroimaging. 2015;25(6):875-82.

8. Witwer BP, Moftakhar R, Hasan KM, Deshmukh P, Haughton V, Field A, et al. Diffusion-tensor imaging of white matter tracts in patients with cerebral neoplasm. J Neurosurg. 2002;97(3):568-75.

9. Romano A, Ferrante M, Cipriani V, Fasoli F, Ferrante L, D'Andrea $G$, et al. Role of magnetic resonance tractography in the preoperative planning and intraoperative assessment of patients with intra-axial brain tumours. Radiol Med. 2007;112(6):906-20.

10. Dubey A, Kataria R, Sinha VD. Role of Diffusion Tensor Imaging in Brain Tumor Surgery. Asian J Neurosurg. 2018;13(2):302-6.

11. Cho JM, Kim EH, Kim J, Lee SK, Kim SH, Lee KS, et al. Clinical use of diffusion tensor image-merged functional neuronavigation for brain tumor surgeries: review of preoperative, intraoperative, and postoperative data for 123 cases. Yonsei Med J. 2014;55(5):1303-9.

12. Costabile JD, Alaswad E, D'Souza S, Thompson JA, Ormond DR. Current Applications of Diffusion Tensor Imaging and Tractography in Intracranial Tumor Resection. Front Oncol. 2019;9:426.

13. Kono K, Inoue Y, Nakayama K, Shakudo M, Morino M, Ohata $\mathrm{K}$, et al. The role of diffusion-weighted imaging in patients with brain tumors. AJNR Am J Neuroradiol. 2001;22(6):1081-8.

14. Salama GR, Heier LA, Patel P, Ramakrishna R, Magge $\mathrm{R}$, Tsiouris AJ. Diffusion Weighted/Tensor Imaging, Functional MRI and Perfusion Weighted Imaging in Glioblastoma-Foundations and Future. Front Neurol. 2017;8:660

15. Khan KA, Jain SK, Sinha VD, Sinha J. Preoperative Diffusion Tensor Imaging: A Landmark Modality for Predicting the Outcome and Characterization of Supratentorial Intra-Axial Brain Tumors. World Neurosurg. 2019.

16. Castellano A, Bello L, Michelozzi C, Gallucci M,
Fava E, Iadanza A, et al. Role of diffusion tensor magnetic resonance tractography in predicting the extent of resection in glioma surgery. Neuro Oncol. 2012;14(2):192-202.

Source of Support: Nil; Conflict of Interest: None

Submitted: 14-09-2019; Accepted: 09-10-2019; Published online: 20-10-2019 